\title{
DISTRIBUTIVES, QUANTIFIERS AND A MULTIPLICITY OF EVENTS
}

\author{
Lesley Stirling \\ School of Epistemics \& Department of Linguistics \\ University of Edinburgh \\ 2 Buccleuch Place \\ Edinburgh EH8 $9 L W, U . K$.
}

\section{ABSTRACT}

With the intention of indicating some temporal/event-theoretic characteristics of distributive clauses, a generalisation is made over distributives and clauses marked for iterative aspect: two kinds of semantic phenomena which have normally been confined to separate theoretical domains. It is shown that in particular, both give rise to an inferential set construction' problem. An informal outline is given of what might constitute such a generalisation. The generalisation is proposed intially on grounds of prima facie plausibility, but its ultimate defensibility and explanatory value will depend on the validity of its consequence, that distributive clauses entail a multiplicity of temporal entities or events. This proposal is considered with respect to two types of discourse phenomena; anaphoric reference to event entities, and temporal binding. These provide further support for making the generalisation, clarify its nature and indicate in what respect the entailment claim can be true of distributives. The set construction problem is of practical importance for computational models of natural language interaction, and since the concept of iterated action is central to planning, the generalisation across iteration and distributives, along with the observations about their nature, have interesting implications for work in this area.

\section{DISTRIBUTIVES}

First, three points about the body of phenomena called 'distributives'. These are taken to be relatively uncontroversial, and are simply assumed in the rest of this paper.

(i) Examples (a) and (b) in (1) illustrate the distributive/collective distinction. Essentially, sentences containing at least one plural or universaliy quantified NP, the denotation of which might be regarded as a set, can either be read in terms of the individual members of this set (as in 1a), or given a 'group' reading in terms of the set as a whole (as in 1b).

(1)a The three girls each had a pizza. (dist)

b The three girls shared a pizza. (coll)

(ii) It is distributive readings which give rise to the possibility of quantifier scope ambiguities - including interpretations which involve dependency relations between the NPs in the sentence. Dependency interpretations in turn allow 'inferential set construction'. Consider example (2).

\section{(2) Mary gave each boy a book}

a ... and told him to look after it.

b They took them into the garden to read.

On the most common reading of the distributive clause Mary gave each boy a book, there is a dependency relation between the two object NPs such that a book is in the scope of each boy and the interpretation of the former is relative to that of the latter. (2b) illustrates one type of anaphoric relation which distributive clauses may participate in: plural pronouns and definite NPs are used to refer to implicit sets of entities which instantiate variables introduced in distributive clauses by just such singular indefinite NPs in the scope of distributive NPs. So in (2b) the underlined NP they refers to the set of books such that Mary gave each one to some boy.

As Webber $(1979,1983)$ has pointed out, this anaphoric possibility represents a practical problem for computational accounts of discourse, since a mechanism is required for constructing as a 
discourse entity a set with the correct description, on the basis of the semantic representation of the initial distributive sentence containing the singular indefinite NP.

Notice that singular anaphora (as in 2a) is also possible: the singular anaphor indicates the maintenance of a rhetorical mode of 'generalisation through singularisation', which is established by the initial distributive sentence. The pronoun it acts as a placeholder, signifying a representative member of the set of books such that Mary gave each one to some boy, and it has the status of a 'bound variable'. Such pronouns cannot be taken to refer and I describe the contrast between them and the plural anaphors as being that the plural ones represent a 'referential' or 'extensional' cashing out of the initial semantic value.

(iii) Sentence (2b) also indicates the possibility of using a plural pronoun they - to refer to some relevant set of boys (in contrast to (2a) where the singular, 'bound variable' pronoun him is used). However, it is not really necessary to propose a second algorithm to construct such sets because the initial, syntactically singular distributive NPs, such as each boy, are arguably almost always themselves 'referential' and 'anaphoric', just in the sense that the range of quantification is restricted by context. Here, the quantifier does not range over the set of all boys, but over some otherwise specified subset ['host' or 'witness set' - Barwise \& Cooper, 1981] of boys. It can be paraphrased with the partitive expression: each of the boys.

My intention in this paper is to explore a temporal, event-oriented perspective on distributives. The question at issue is: what are the consequences for the temporal or eventtheoretical analysis of a clause, of its being 'distributive' in the sense defined?

First I indicate similarities between distributives and clauses marked with iterative aspect. I then make certain observations which apply to both distributives and iteratives, concerning two kinds of discourse phenomena: definite NP anaphora to event entities, and temporal binding. These support the hypothesised generalisation and tell us something about the temporal or eventtheoretical structure of both distributives and iteratives.

The observations made in this paper are confined to the past tense and to 'telic' or 'bounded' situation types (Mourelatos, 1981).

\section{ITERATIVES}

The following are examples of iterative clauses in English:

(3) a Each day Mary wrote a letter to her sister.

b Every time John went abroad he bought a souvenir.

c Vesuvius erupted three
times.

Iterativity is an aspectual parameter which in English is marked mainly adverbially, with the presence of what are called 'frequency adverbials' or 'temporal quantifiers' (underlined in the examples). Iterative markers on a clause indicate the repetition of the event described in the clause, and also provide information about the frequency of repetition. The key requirement is couched in terms of an entailment condition: iteratives entail that there was more than one occasion on each of which an event of type E occurred, where 'occasion' is defined as spatiotemporal location. So there is a necessary requirement of sequentiality: that the events in question occurred on different occasions, in sequence.

\section{GENERALISATION}

On the basis of the descriptions given in sections 1 and 2 we can argue for $a$ generalisation over distributives and iteratives such as is represented in Figure 1 (cf. Forsyth, 1970: 154).
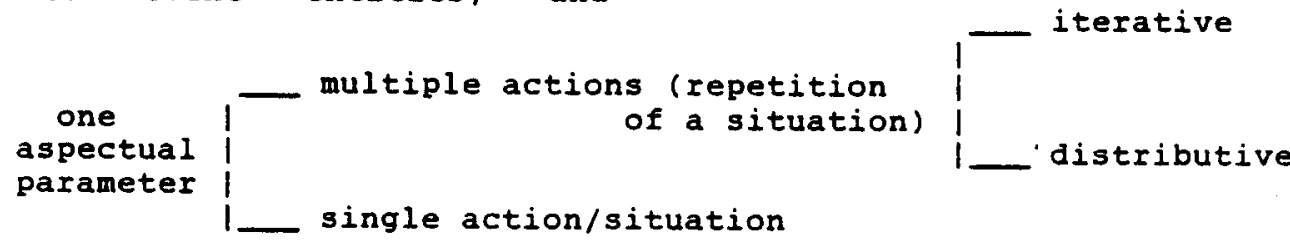

Figure 1. Generalisation over distributives and iteratives. 
There are a number of intuitive arguments for the prima facie plausibility of such a generalisation, two of which will be considered here.

First, it is clear that from one point of view, distributives and iteratives differ just in the nature of what is quantified over: distributives, where the marking of repetition is normally on the NP, involve quantification over individuals, while the temporal adverbial expressions in the iterative examples are normally taken to involve quantification over times or events. That is, the iterative markers can be seen as introducing distributive quantification.

secondly, perhaps the strongest argument for generalisation is that inferential set construction occurs with iteratives. Some iteratives, like distributives, contain indefinite NPs whose instantiations may vary across the repetition of the event. $A$ souvenir in example (4), and a letter in example (5) are just such NPs.

(4) Everytime John went abroad he bought a souvenir.

a Sometimes it was a silver teaspoon, sometimes an antique vase.

b They just lie around the house, cluttering it up.

(5) Each day, Mary wrote a letter to her sister.

a It was usually ten pages long.

b They were chatty letters.

Iteratives such as these manifest the same scope ambiguities as distributives and are open to the same mechanism of 'set construction' which as we saw operates in distributive clauses on the NPs which are distributed over. So in (4), a souvenir is in the scope of a restricted universal quantification over times, and the sentence in question could be followed by (b) where they refers to the set of souvenirs such that John bought each one on some trip abroad. (4a) and (5a) show that something like the 'bound variable' anaphora of example (2a) is possible here too. [1]

[1] The expression 'variable binding', traditionally taken to describe an intrasentential phenomenon, is used with a less restrictive sense in this paper.
The solution to the set construction problem should if possible be a general one, covering both distributive and iterative cases.

The ultimate defensibility, and explanatory value, of making such a generalisation will depend on whether it accurately reflects the facts about the temporal or event-theoretical structure of distributive clauses. It was said that iteratives are customarily defined as entailing a sequence of events, each associated with a different occasion or spatiotemporal location. A generalisation of the kind envisaged would suggest that distributives might be regarded as having such an entailment as well, and the question we have to consider is whether this is so.

Certainly, some distributives clearly involve multiple occasions, that is, spatiotemporal locations. For example (6a), where it's one and the same book which is lent, and (6b).

(6) a Mary lent each boy the book.

b John visited each of his

On the other hand, take an example like (2) above (Mary gave each boy a book). This could easily describe a single occasion of giving, taking place once only. One could even imagine Mary handing a book to each of two boys simultaneously, with either hand. Or consider (7), on the interpretation where it was the same message and $I$ did it on one occasion.

(7) Yesterday I sent each student a message via computer mail.

Intuition is clearly not enough, and in sections 4 and 5 we shall consider evidence from the discourse phenomena mentioned earlier, but first, what would constitute such a generalisation over iteratives and distributives: a generalisation which would indicate their similarities and differences, and provide a way in which the entailment of multiple temporal entities/events could be said to be true of distributives? 


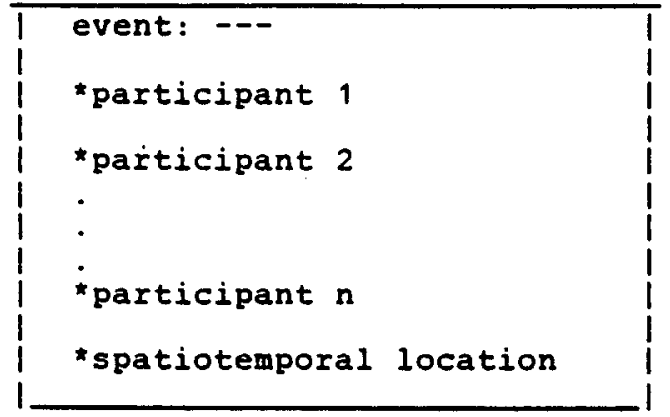

Figure 2. 'Case' specification

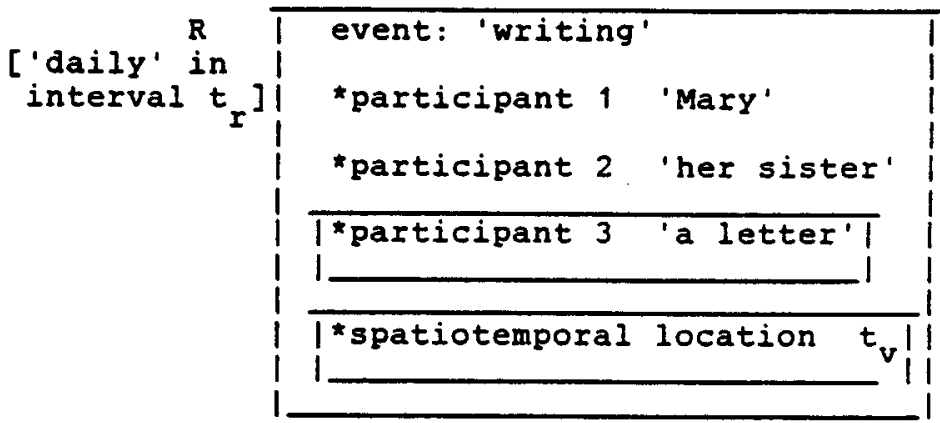

Figure 3. Case specification for Each day, Mary wrote a letter to her sister

We can regard a clause as providing, at one level of description, a pattern or template, with specification of an event type with respect to placeholders for the following elements: one or more participants, and spatiotemporal location. Figure 2 is the skeleton of such templates or 'case specifications', which will be fleshed out for individual clauses. Distributive and iterative markers alike then contribute the following information:

- first, that there are multiple instantiations of this pattern rather than a single instantiation:

- and, in conjunction with other grammatical features of the clause, they provide information about which of these elements remain constant across such instantiations and which are variable.

For example, take the sentence Each day, Mary wrote a letter to her sister (Figure 3). This specifies a writing event type which (as indicated by ' $R$ ') is repeatedly instantiated. Two of the participants, Mary and her sister, remain constant across these repetitions. The other participant, the letter, varies and will be instantiated by a (possibly) different entity on each occasion of repetition. There is also variation in the spatiotemporal element. This element is represented here by the time variable, $t$, while $t$ represents the reference time of the iterated complex as a whole.

It is with respect to this second kind of information, about which of the elements vary, that iteratives and distributives differ. In iterative but not in distributive clauses the spatiotemporal location will necessarily be among those elements which are variable; other elements may also vary if they are introduced by expressions in a scopal dependency relation with the temporal quantifier phrase. In

[2] 'Case' in the sense of Lewis (1975) rather than Fillmorean Case Gram$\operatorname{mar}$.

distributives, at least one of the participant elements must vary - the one introduced by the distributive NP - and in addition so may other elements, including spatiotemporal location, if they are in a dependency relation with this NP. If no other participant element is in a dependency relation with the distributive NP, that is if all other participant elements are held constant, then variation in spatiotemporal location will entail, as in example (6a). Which elements vary tells us which inferential sets to construct.

The similarities between the informal proposal which has just been sketched and Lewis's (1975) account of temporal quantifiers in terms of cases will be obvious. Lewis defines a case as an $n$ tuple of its participants (i.e. the values of free variables in the sentence) and $a$ time coordinate [the 'case specifications' represented above include in addition an event type label, such as 'writing']. $\mathrm{He}$ argues that iterative markers involve quantification over cases rather than over times or events. My proposal could be interpreted as a claim that we can extend some version of a case analysis to include distributives: these, too, would be taken to involve quantification over cases. Quantification over times or participants would then represent particular ways of realising the multiple instantiation of the case. The advantages of an account such as this are that it allows generalisation over a wide variety of phenomena, including numerous ways of marking iteration or distribution on the clause, and that it provides an appropriate level of description to be referred to in accounting for discourse anaphoric relations.

The question of the individuation of events is a controversial one, and the terms 'event' and 'event type' have so far been used rather loosely. Suppose we first follow Mourelatos (1981) in making a distinction between events and the spatiotemporal locations with which they are associated, and secondly, suggest that 
events should be regarded as particular instantiations of case specifications of the type described. Then iteratives entail a multiplicity of cases/events + spatiotemporal locations (which are in some isomorphic relationship), whereas distributives likewise entail a multiplicity of cases/events, but merely allow the possibility of multiple spatiotemporal locations (i.e., events and spatiotemporal locations may not be in an isomorphic relationship) - as we shall see, in the case of any particular distributive, this question may be resolved by following anaphoric reference.

\section{ANAPHORIC REFERENCE TO EVENTS}

(8) John visited each of his great aunts.

a The visits were much appreciated by the old ladies.

b These occasions were a source of torment to him.

(9) Everytime John went to Namibia he visited an old friend.

a He found the visits distressing.

b He was upset on these eccasions.

Examples ( 8 ) and (9) indicate that after a distributive or iterative clause, speakers can refer using a plural definite NP, to a set of entities which are something like 'events' or 'occasions' and which appear to correspond to the distributive or iterated situation of the preceding clause. Note the two types of example ( $a$ and $b$ ): in the first, the common noun head of the anaphoric expression is a nominalisation of the verb in the antecedent sentence (such NPs are normally taken to refer to event entities): in the second the noun is a much more general one. Both kinds of example appear to represent a phenomenon of the same type as the inferential set construction described in section 1: it is as if the initial distributive or iterative clause introduces an 'event' variable and the anaphoric NPs represent a referential/extensional cashing out of this.

Continuing the parallel with anaphoric reference to nominally-introduced entities, example (10) shows that with respect to event entities too, singular anaphoric NPs occur which are of the 'bound variable' type. Thus in (10) the visit signifies a representative event of the type described:

(10) John visited each of his maiden aunts, but the visit didn't make him popular with any of them.

A second type of singular anaphora occurs, as in (11): in this case the anaphoric pronoun that appears to refer to the whole distributive episode, of John visiting each aunt, described in the preceding clause.

(11) John visited each of his aunts. That was a thoughtful thing to do.

Finally, examples (12) and (13) show that, although plural definite NPs can be used to access the set of events or occasions resulting from distributive/iterative clauses (as in (8) and (9)), plural pronouns cannot. In (12) they and them can only be interpreted with reference to the aunts - compare the same sentences with the pronouns replaced by the NP the visits. Explanation of this, which there is not space to elaborate here, must take account of the obvious fact that a requirement of nominalisation is involved. Meanwhile the important point to note is the clear distinction between the distribution of definite NP and pronominal anaphors.

(12) John visited each of his aunts.

a * They were a dismal failure.

$b$ * He had a good time on all of them.

c * Each of them went well.

(13) John flew to Paris twice.

a He enjoyed both trips.

b * He enjoyed both of them.

Example (11) shows that singular pronominal reference does occur, but this seeming inconsistency may be explained in terms of the nature of the entity being referred to: the plural NPs seem to refer to events whereas the singular ones refer to 'facts' or 'propositions' (in the sense of the distinction argued for in vendler $(1967))$. [3]

[3] There is clearly much more to be 


\section{TEMPORAI BINDING PHENOMENA}

First it is necessary to review some claims of theories of temporal reference.

Consider the following example:

(14) when John made the cake, he broke the oven.

When, like other 'temporal connectives' such as before and after, indicates a particular relationship between the two clauses it connects: normally such relationships are considered to consist in specification of the relative ordering in time of the two events described by these clauses. The basic claim, then, is that such connectives give a specification of a kind of 'temporal binding' between the two clauses such that the reference time of one is dependent on that of the other. This claim is independent of the particular nature of the ordering or binding hypothesised. (This idea is clearly on a par with claims that certain temporal phenomena should be understood on analogy with nominal anaphora, for example that the (past) tense morpheme should be taken to refer to a time, and to receive its reference either from some antecedent tense morpheme in the text, or from some adverbial expression, or deictically.)

Kamp (1979), Hinrichs (1982) and Partee (1984), who subscribe to a discourse theory of temporal (ordering) relations, argue that (in narrative discourse) the reference time of any main clause will be dependent on that of the preceding clause; that is, that just the same kinds of temporal binding dependencies occur at the level of discourse, between clauses which are syntactically independent as well as between those which are linked by temporal connectives.

With this background, we can consider clauses which involve iteration, marked by temporal quantification, in addition to temporal connection of the type just described. For example (15).

(15) When Max left the office, he always turned the lights off.

It seems that such sentences

said with respect to the phenomena examined in this section; this will be dealt with in forthcoming work. constitute a binding or dependency, between two clauses describing distinct types of event, which puts a condition on the pairwise mapping of instantiations of such events. Thus, in this case, every event of Max leaving the office is said to have been paired with (at least one) event of his turning the lights off. This kind of dependency relation seems to be exactly parallel to that which holds between the distributive NP each girl and the indefinite NP a book in Mary gave each girl a book. In terms of the schema in Figures 2 and 3 , binding is of the temporal variable $t$ of clause one, and the $t_{v}$ of clause two.

Notice that the when clause restricts the range of the iterative quantifier always in the following clause. Examples like (15) are a special case of temporal quantification in that the condition on the range of quantification is explicitly stated in the subordinate clause. But it is true in general that temporal quantifiers like always or each time do not range over all of time, but over some contextually fixed interval. They thus conform to the claim made in section 1 that distributive NPs normaliy have their range of quantification restricted to some otherwise-specified 'witness set'. The reference time $t$ of Figure 3 represents this kind of witness set.

The main point to be made in this section is that distributive clauses, with no explicit temporal quantification, can also act as sources for temporal binding, and restrict the range of subsequent temporal quantification, in just the same way that the iterative clauses do. Hence we find examples like (16) and (17).

(16) Mary gave each boy a book. Sometimes the boy thanked her, but more often he did not.

(17) Harry invited each of his relatives to stay. On each occasion he bought in enough food to feed an army.

Here, the range of the quantifier phrases sometimes, more often and on each eccasion is restricted by the set of events determined by the preceding distributive clause - that is, by something like the set of instantiations of the case specification, perhaps more specifically, instantiations of the temporal variable $t$. There is binding between the eventy described in the two sentences: in (16) some of the 'times' (occasions) on which Mary gave some boy a 
book are such that the boy in question thanked her; in (17) each occasion on which Harryis relatives came to stay is paired with an event of his buying in lots of food.

Example (18), which is from a university library photocopying machine, is exactly paraliel to the temporally quantified and connected example given in (15). Here, each plays the same role as always does in (15).

(18) Please wait until machine is completely silent before inserting each 5p coin.

This example is (theoretically) ambiguous in the scope of the distributive marker: does the first clause represent a condition for the iterated event, so that we are to wait until the machine is silent and then insert all the $5 \mathrm{ps}$ one after another, or is it part of an iterated condition + event sequence, so that on each occasion of inserting $5 p$, we must wait until the machine is silent first? (In actuality, it is of course the second reading which is intended.)

Now consider the following examples, with another temporal connective, then.

\section{(19) Mary gave each boy a book.}

a Then she marked his name off her list as having received it.

b Then she dismissed them.

(20) Everytime John went abroad he brought back a souvenir.

a Then he put it in the cupboard with the rest of them.

$b$ Then he stopped going abroad and threw them all away.

These examples indicate that the initial distributive or iterative sentence can be viewed either in terms of its unity as a complex situation, or in terms of the constituent phases which that total comprises (cf. examples (8) and (11) in section 4). Hence in (19) the reference time of (b), the dismissal, will be after the completion of the complex event of giving out books, (in terms of the case specification in Figures 2 and 3 the $t$ of (b) will be after the $t$ of the initial sentence). In contrast, the sequence in (19a) is such that the then clause is 'in the scope of 'the distributive and there is a pairwise binding between the event type of giving out $a$ book and the event type of marking off a name, such that each instantiation of the former will be followed by an instantiation of the latter. That is, there is a temporal ordering condition on the temporal variables $t$ of the two clauses. The whole sequence will have the same $t_{r}$; overall reference time or 'witness set'. If we regard temporal connectives as indicating a relationship defined over reference times, then we are put in the position of recognising two possibilities with respect to distributive/iterative sentences: these seem to involve something like 'nested reference times', with the ordering relation being able to hold over either $t_{v}$ or $t_{r}$. Consider also examples (21) and (22).

(21) Mary gave each boy a book.

a On each occasion the boy thanked her.

$b$ on that occasion they were grateful to her.

(22) John visited each of his maiden aunts. Each one gave him a cup of tea.

Example (22) is particularly interesting, because we have a sequence of two clauses which are not syntactically linked by a temporal connective, and neither of which contains a temporal quantifier phrase. Yet there is an implication that there is a pairwise dependency relation between teas and visits. The generalised binding relationship appears to hold across the sentence boundary, just as Kamp, Hinrichs and Partee postulated with respect to ordering relations between clauses describing single rather than repeated situations. Any analysis of this discourse which assigns each sentence a single reference time, and which then, following Hinrichs and partee, requires that the reference time for the second follow that of the first, will give the wrong results. [4]

The observations which have been made evince a close analogy with the facts of

[4] Their response to this might be that we are not here dealing with narrative discourse, a denial which is more plausibly made of earlier examples, or even (19) or (20), than it is of (22). 
nominal anaphora which were outlined in section 1 of the paper. The (a) examples in (19), (20) and (21) in each case represent something like maintenance of the binding: we are still specifying the type of the event sequence which is iterated, and all the clauses in the sequence have the same $t$; reference time or witness set. In the ( $b$ ) examples, on the other hand, the description of the iterated event is finished: there is a discourse-level division between the initial distributive/iterative sentence and sentence (b), representing the end of the rhetorical mode of generalisationthrough-singularisation, and closure of the interval $t$. One indication of this is that any pFonouns occurring in the (b) sentence, and anaphoric to distributive NPS or their dependent expressions in the first sentence, will necessarily be plural. The use of a plural NP, which as I have said represents referential cashing out', indicates that the iterated sequence is over and we can now assume its completion and move on. In contrast, in the (a) sentences anaphoric pronouns referring to entities which were introduced by the nominals in the distributive/iterative sentence, are normally singular.

\section{CONCLUSIONS}

The anaphoric examples in section 4 show that both distributive and iterative clauses enable subsequent reference to sets of cases/events using a definite NP. This is clearly paraliel to the possibility of using a plural anaphoric NP to make reference to some set of individuals (such as books), on the basis of an original singular NP introduction. It would seem to be desirable to account for both kinds in terms of the same mechanism of 'set construction'. The anaphoric examples also indicate a qualitative difference between the distribution of definite NP and pronominal anaphora, and show that there is discourse anaphoric access not just to the subparts of the distributed or iterated situation, but also to something like the whole distributive quantification, which may be an entity of a different kind.

\footnotetext{
The temporal binding phenomena of section 5 give a different type of evidence for the explanatory value of making a generalisation of the kind described. The observations in this section also show that, whereas past tense clauses describing a single situation have a reference time with some specific temporal referent, distributive and iterative clauses seem to require 'nested
}

reference times', with temporal ordering relations definable at two levels. The Kamp/Hinrichs/Partee account needs to be modified before it is able to incorporate these phenomena.

I've said that the set construction problem is of practical importance for computational models of natural language interaction. In addition, the concept of iterated action is important to planning, so that a generalisation across distributives and iteratives plus what has been said about their temporal nature should have interesting implications in this area. If iteration is handled computationally by setting up a loop which embodies the instruction to repeat an action under certain conditions, then distributives may be handled the same way. Distributive and iterative interpretations may hold over stretches of discourse, the delimitation of which is relevant to the interpretation of temporal connectives, and as we saw with examples (19) to (21), in some cases anaphoric phenomena may give clues about this delimitation, that is, indicating when to turn off the iterative loop. The delimitation of such discourse chunks corresponds to the delimitation of the extent of influence of the $t$ or 'witness set', and so anaphora in following sentences may allow us to close off this interval.

\section{ACKNOWLEDGEMENTS}

This paper results from research towards a PhD which has been supported by a grant from the Association of Commonwealth Universities. The development of the ideas presented here has benefitted considerably from the opportunities for stimulating discussion provided by the School of Epistemics Tense and Discourse Workshop. I am particularly grateful to the following people for helpful comment on earlier versions of the paper: Mark Steedman, Marc Moens, Henk Zeevat, Ewan Klein, Han Reichgelt, Barry Richards, Jim Miller, Jan de Vuyst, David McCarty, Jim Hurford. 
Barwise, Jon \& Cooper, Robin (1981) 'Generalised quantifiers and natural language' Linquistics and Philosophy 4, 159-219.

Forsyth, J. (1970) A grammar of aspect. C. D.P.

Hinrichs, Erhard (1982) 'Temporal anaphora in discourses of English' Unpublished.

Kamp, Hans (1979) 'Events, instants and temporal reference' in Baeuerle et al (eds) semantics from different points of view. Berlin: springer, 277-322.

Lewis, David (1975) 'Adverbs of quantification' in E.L. Keenan (ed) Eormal semantics of natural lanquaqe. C.U.P., 3-15.

Mourelatos, Alexander P.D. (1981) 'Events, processes and states' in syntax and semantics vol 14: Tense and aspect. N.Y.: Academic Press, 191-212.

Partee, Barbara Hall (1984) 'Nominal and temporal anaphora' Iinquistics and Philosophy 7, $243-86$.

Vendler, zeno (1967) Linquistics in philosophy. Ithaca: Cornell U.P.

Webber, Bonnie Iynn (1979) A formal approach to discourse analysis. The Hague: Mouton.

Webber, Bonnie Lynn (1983) 'So what can we talk about next?' in $M$. Brady and $R . C$. Berwick (eds) Computational models of discourse. Cambridge, Mass.: MIT Press, $331-71$. 